\title{
Saturation of the width of the strength function*
}

\author{
A.J. Sargeant, M.S. Hussein, M.P. Pato and M. Ueda \\ Nuclear Theory and Elementary Particle Phenomenology Group, Instituto de Física, \\ Universidade de São Paulo, C.P. 66318, 05315-970 São Paulo, SP, Brazil
}

(September 6, 2018)

\begin{abstract}
The strength function of a single state $|d\rangle$ is studied using the deformed Gaussian orthogonal ensemble. In particular we study the dependence of the spreading width of $|d\rangle$ on the degree of mixing.
\end{abstract}

The mixing of a single state with a background of complicated states is important in the description of a variety of phenomena in nuclear physics such as isobaric analogue resonances, giant dipole resonances and the decay out superdeformed rotational bands. Such can mixing be conveniently described by the strength function [] and it is interesting to study the generic features of this object. Previous studies in this vein have investigated a single state coupled to a background generated by a two-dimensional anharmonic oscillator [2] and the spreading of a shell model basis state over the shell model eigenstates due to the the residual interaction [3]. Here we use random matrix theory [4].

We write the Hamiltonian, $\mathrm{H}$, as the sum of two terms,

$$
H=H_{0}+\mathcal{V} .
$$

The eigenstates, $|n\rangle$, and eigenvalues, $E_{n}$, of $H$ satisfy

$$
H|n\rangle=E_{n}|n\rangle, \quad n=1, \ldots N+1,
$$

whilst for $H_{0}$ we have

$$
\begin{aligned}
& H_{0}|k\rangle=E_{k}|k\rangle, \quad k=1, \ldots N, \\
& H_{0}|d\rangle=E_{d}|d\rangle, \quad d=N+1 .
\end{aligned}
$$

The strength function is then defined as

$$
F_{d}(E)=\sum_{n=1}^{N+1}|\langle d \mid n\rangle|^{2} \delta\left(E-E_{n}\right),
$$

${ }^{*}$ Supported in part by FAPESP. 
and describes how the state $|d\rangle$ is distributed over the $N+1$ eigenstates of $H$. We shall choose the energy of $|d\rangle, E_{d}$, such that it lies in the middle of the the spectrum of $H$.

Some insight into the behaviour of the strength function can be obtained by performing a two step diagonalisation of $H$ [1]. Let us represent the Hamiltonian in the basis $\{|k\rangle,|d\rangle\}$. Diagonalising $H$ in the $N$-dimensional subspace defined by excluding $|d\rangle$ we obtain the set of eigenvectors $|q\rangle=\sum_{k}\langle k \mid q\rangle|k\rangle$ with eigenvalues $E_{q}, q=1, \ldots, N$. The Hamiltonian in the basis $\{|q\rangle,|d\rangle\}$ has diagonal matrix elements $E_{q}, q=1, \ldots, N$ and $E_{d}, d=N+1$ and nonzero off-diagonal elements $\mathcal{V}_{d q}=\mathcal{V}_{q d}=\sum_{k} \mathcal{V}_{d k}\langle k \mid q\rangle$ (the Hamiltonian is assumed to be real symmetric). The diagonalisation of the intermediate matrix may be carried out analytically

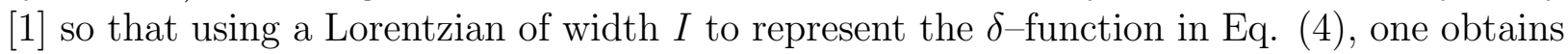
《1]

$$
F_{d}(E)=\frac{1}{2 \pi} \frac{\Gamma_{d}^{\downarrow}+I}{\left(E-E_{d}-\Delta_{d}^{\downarrow}\right)^{2}+\left(\frac{\Gamma_{d}^{\downarrow}+I}{2}\right)^{2}}
$$

where

$$
\Gamma_{d}^{\downarrow}(E)=I \sum_{q} \frac{\left|\mathcal{V}_{d q}\right|^{2}}{\left(E-E_{q}\right)^{2}+\left(\frac{I}{2}\right)^{2}}
$$

and

$$
\Delta_{d}^{\downarrow}(E)=\sum_{q} \frac{\left|\mathcal{V}_{d q}\right|^{2}\left(E-E_{q}\right)}{\left(E-E_{q}\right)^{2}+\left(\frac{I}{2}\right)^{2}} .
$$

By making the further assumptions that the eigenvalues $E_{q}$ are equi-distant with mean spacing $D$, that the squared matrix elements $\left|\mathcal{V}_{q d}\right|^{2}$ have approximately the same order of magnitude, $\left\langle\mathcal{V}^{2}\right\rangle$, for all $q$ and that the magnitude of $\sqrt{\left\langle\mathcal{V}^{2}\right\rangle}$ is smaller than the energy range in which it may be considered constant (whilst being larger than $D$ in order for the strength function to have meaning) the strength function may be approximated by a Lorentzian [1, 3]

$$
F_{d}(E) \approx \frac{1}{2 \pi} \frac{\Gamma_{d}^{\downarrow}}{\left(E-E_{d}\right)^{2}+\left(\frac{\Gamma_{d}^{\downarrow}}{2}\right)^{2}}
$$

where the spreading width is given by the "golden rule"

$$
\Gamma_{d}^{\downarrow} \approx 2 \pi \frac{\left\langle\mathcal{V}^{2}\right\rangle}{D}
$$

We wish to study how the distribution, $F_{d}(E)$, depends on the degree of mixing by which we mean the strength of $\mathcal{V}$. To that end we employ deformed Gaussian orthogonal ensemble (DGOE) [5]. In this model $H$ is real symmetric and it's matrix elements are taken to be independent Gaussian distributed random numbers with zero mean and variances $\left\langle\left(H_{0}\right)_{k, k}^{2}\right\rangle=\frac{a^{2}}{2 N}$ for the diagonal matrix elements and $\left\langle(\mathcal{V})_{k, k^{\prime}}^{2}\right\rangle=\frac{\lambda^{2} a^{2}}{4 N}$ for the off-diagonal matrix elements. We take $\left\langle(\mathcal{V})_{k, d}^{2}\right\rangle=\left\langle(\mathcal{V})_{d, k}^{2}\right\rangle=\frac{\lambda^{2} a^{2}}{4 N}$ as well although choosing a different variance for this matrix element may be appropriate in some applications. 
The parameter $\lambda$ may be varied between 0 and 1 and determines the degree of mixing. The parameter $a$ determines the energy interval over which the eigenvalues are distributed. In the limiting case $\lambda=0$ the eigenvalues $E_{n}$ are Gaussian distributed;

$$
\rho_{0}(E)=\frac{N^{\frac{3}{2}}}{a \sqrt{\pi}} \exp \left(\frac{-N E^{2}}{a^{2}}\right),
$$

whilst in the limit $\lambda=1$ they are distributed according to the Wigner semicircular law;

$$
\rho_{1}(E)=N \frac{2}{\pi a^{2}} \sqrt{a^{2}-E^{2}} .
$$

We perform an unfolding of the spectrum of the spectrum of $H$ defined by

$$
x_{n}=\int_{-\infty}^{E_{n}} d E \rho(E),
$$

where $\rho(E)$ is the smoothly varying part of the level density. Thus the unfolded spectrum has mean level density equal to 1 and is dimensionless. The smooth variation the level density was obtained in practice by fitting the cumulative level density (staircase function)

$$
x=\sum_{n=1}^{N+1} \theta\left(E-E_{n}\right)
$$

(the letter $\theta$ denotes the unit step function) to a polynomial using the method of linear least squares [6]. For the case $N=50$ (used in the calculations below) we found the function which gave the best visual fit was a polynomial of degree 5 .

In order to compare our calculation of the strength function on the unfolded energy scale with the Lorentzian approximation, Eqs. (8) and (9), we apply a simplified unfolding to these approximate formulae, defined by

$$
x=\frac{E}{D} .
$$

Thus the unfolded version of the golden rule is

$$
\frac{\Gamma_{d}^{\downarrow}}{D} \approx 2 \pi \frac{\left\langle\mathcal{V}^{2}\right\rangle}{D^{2}} .
$$

Persson and Ȧberg [7] proposed the following formula for the mean (meaning averaged over the energy range where the eigenvalues are distributed) density of states of the DGOE which interpolates between $\lambda=0$ and $\lambda=1$, valid when $a=2$ :

$$
\bar{\rho}_{\lambda}=\frac{N^{\frac{3}{2}}}{4 \lambda N^{\frac{1}{2}}+7 N^{-1.5 \lambda}} .
$$




\section{FIGURES}
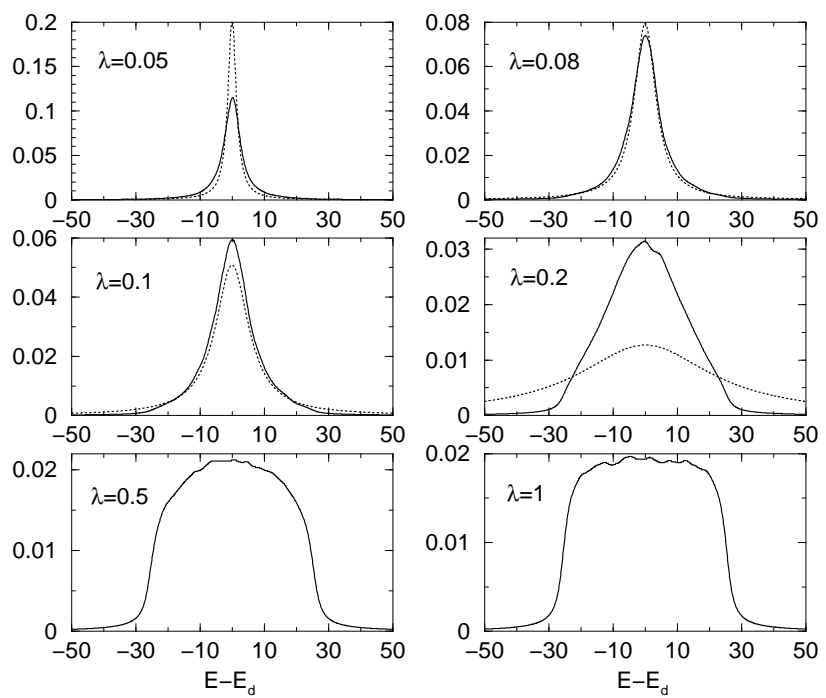

FIG. 1. Strength function, $F_{d}(E)$, for various values of the mixing parameter, $\lambda$, calculated using DGOE (solid lines) and calculated using the Lorentzian approximation (dotted lines). See text for discussion.

Figure 11 displays our calcuations of the strength function (solid lines) for various $\lambda$ using Eqs. (5- 6) where the eigenvalues (unfolded using the procedure defined by Eq. (12) ) and eigenvectors are generated by the DGOE. We performed our calculations using $N=50$ whilst the width of the the Lorentzian used to represent the $\delta$-function (Eq. (国)) is $I=3$. We set $a=2$.

The dotted lines in fig. 11 are the strength function calculated using the Lorentzian approximation, Eqs. (8) and (9). The mean square value of the coupling is taken to be $\left\langle\mathcal{V}^{2}\right\rangle \approx \frac{\lambda^{2} a^{2}}{4 N}$. The mean level spacing $D$ is taken to be $\frac{1}{\rho_{0}(E=0)}=\frac{a \sqrt{\pi}}{N^{\frac{3}{2}}}$; the lower limit for the level spacing of the DGOE spectra.

Using the estimates of the previous paragraph, the condition $\frac{\left\langle\mathcal{V}^{2}\right\rangle}{D^{2}}>1$ (for the single state $|d\rangle$ to be significantly mixed with more than a single one of the $|q\rangle)$ implies $\lambda>\frac{2 \sqrt{\pi}}{N}$. Thus for $N=50$, although we can calculate the strength function for arbitrary small $\lambda$ it is only meaningful for $\lambda>0.07$. The value of $I$ should be chosen so that it is a negligable fraction of the combined width $\Gamma_{d}^{\downarrow}+I$ whilst being greater than the level spacing (unity after unfolding).

From fig. 11 we can see that for weak $\lambda$ the strength function has an approximately Lorentzian shape. As we decrease $\lambda$ below 0.07 the strength function approaches a Lorentzian whose width is dominated by $I$. For $\lambda>0.07$ the strength function starts to deviate significantly from the Lorentzian shape already at $\lambda=0.1$ broadening towards a semicircle before $\lambda=1$. An ensemble average was performed over 100 realizations for the cases $\lambda=0.05,0.08$ and 0.1 whilst to obtain a relatively smooth strength function for the $\lambda=0.2$, 0.5 and it was necessary average over 1000 realizations. 


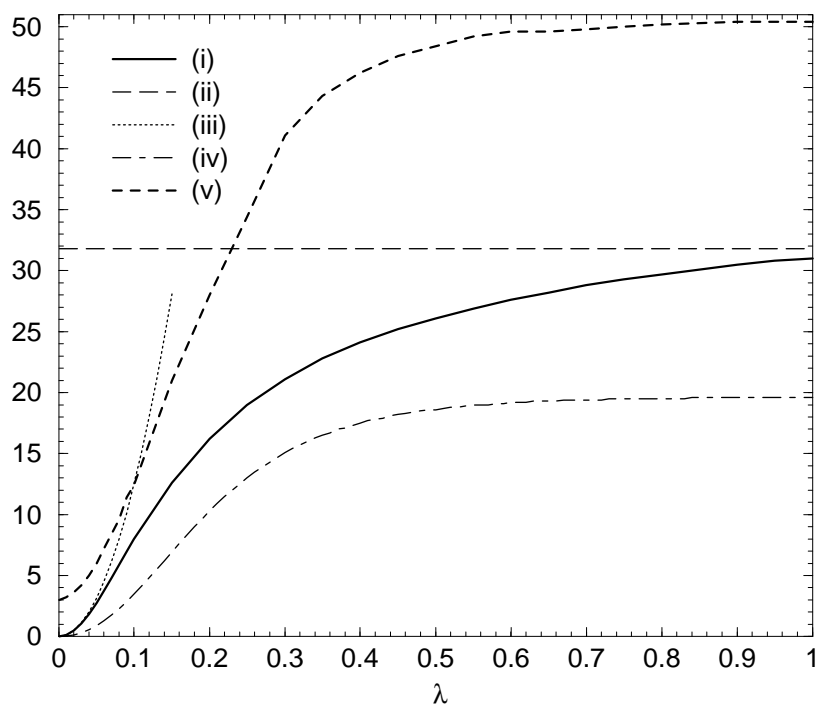

FIG. 2. Spreading width of $|d\rangle$ as a function of the mixing parameter $\lambda$. See text for meaning of (i)-(iv).

In fig. 2 we plot the following calculations for the spreading width of $|d\rangle$ as a function of $\lambda$ :

(i) Eq. (6), at the peak value $E=E_{d}$ where the eigenvalues (unfolded using the procedure defined by Eq. (12)) and eigenvectors are generated by the DGOE. An ensemble average was performed over 100 realisations for all $\lambda$; otherwise the same parameters are used as were used in fig. 11.

(ii) The limiting value $(\lambda=1)$ for the width calculated using the golden rule (Eq. 15) and density Eq. (11]) at $E=0: 2 \pi\left(\frac{a^{2}}{4 N}\right)\left(\frac{2 N}{a \pi}\right)^{2}=\frac{2 N}{\pi}$.

(iii) The golden rule expression for the width using the Poisson density, Eq. (10), at $E=0$ : $2 \pi\left(\frac{\lambda^{2} a^{2}}{4 N}\right)\left(\frac{N^{\frac{3}{2}}}{a \sqrt{\pi}}\right)^{2}=\frac{\lambda^{2} N^{2}}{2}$.

(iv) The golden rule with the density Eq. (16): $2 \pi\left(\frac{\lambda^{2} a^{2}}{4 N}\right) \bar{\rho}_{\lambda}^{2}$.

(v) The full width at half maximum FWHM of the strength function $F_{d}(E)$, Eqs. (5 7), calculated using the DGOE.

Three regions may be identified in the DGOE calculations (lines (i) and (v)) in fig. 2). We see that $\Gamma_{d}^{\downarrow}\left(E_{d}\right)$ for very weak coupling $(\lambda<0.04)$ has a quadratic dependence which accurately follows the golden rule calculation (line (ii)). Between $\lambda=0.05$ and $\lambda=0.15$ the this dependence is linear becoming very weak as $\lambda$ approaches unity. The behaviour of the DGOE is simulated by Eq. (16) of Persson and Äberg for the average level density (line (iv)). At $\lambda=1$ our DGOE calculation of $\Gamma_{d}^{\downarrow}\left(E_{d}\right)$ is approximately a factor $\left(\frac{\rho_{1}(E=0)}{\bar{\rho}_{1}}\right)^{2}=\left(\frac{4}{\pi}\right)^{2}=1.6$ greater than the calculation which employs Eq. (16). The FWHM (line (v)) also has a quadratic dependence on $\lambda$ for weak $\lambda$. A linear dependence is maintained up to $\lambda=0.3$ after which the $\lambda$-dependence becomes very weak as $\lambda$ approaches unity. When $\lambda=0$ the FWHM is just equal to the averaging interval $I(=3)$. In the opposite limit of $\lambda=1$ the FWHM is essentially equal to $N(=50)$; ie. the state $|d\rangle$ is spread over all the eigenstates of $H$. Thus in the limit $\lambda=1$, the golden rule (line $(\mathrm{v})$ ) is a factor $\frac{2}{\pi}$ smaller than the FWHM. Ref. [3] investigated the spreading width of a basis state of a shell model Hamiltonian 
as a function of the strength of the residual interaction (corresponding to $\lambda a$ above). They identified regions where the spreading width has a quadratic dependence on the $\lambda$ (weak mixing) becoming linear for larger $\lambda$. They also found that the golden rule cannot be used to estimate the FWHM of the strength function for strong mixing.

In conclusion we have studied how the shape and width of the the strength function of a single state depends on the degree of mixing, using random matrix theory. An application of these results to the decay out of a superdeformed rotational band is in progress.

A.J.S. acknowledges helpful comments on the manuscript by Professor R.C. Johnson. 


\section{REFERENCES}

[1] A. Bohr and B. Mottelson, Nuclear Structure (Benjamin, NY, 1969), Vol. 1.

[2] H. Aiba, S. Mizutori and T. Suzuki, Phys. Rev. E56 (1997) 119.

[3] N. Frazier, B.A. Brown and V. Zelevinsky, Phys. Rev. C54 (1996) 1665.

[4] T. Guhr, A. Müller- Gröeling and H.A. Weidenmüller, Phys. Rep. 299 (1998) 189.

[5] M.S. Hussein and M.P. Pato, Phys. Rev. Lett. 70 (1993) 1089.

[6] W.H. Press et al., Numerical Recipes in FORTRAN, 2nd ed. (Cambridge Univ. Press, 1994).

[7] P. Persson and S. Ȧberg, Phys. Rev. E52 (1995) 148. 